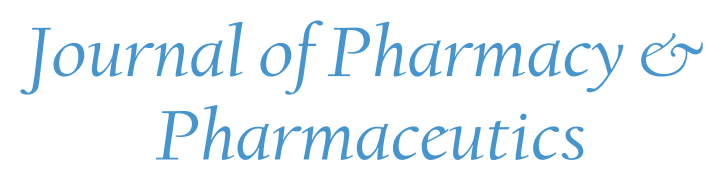

\title{
Bioprospecting for Algal Based Nutraceuticals and High Value Added Compounds
}

\section{Sanjukta Subudhi*}

Environmental and Industrial Biotechnology Division, The Energy and Resources Institute (TERI), Habitat Place, Darbari Seth Block, Lodhi Road, New Delhi 110003, India

*Corresponding author: Sanjukta Subudhi, Environmental and Industrial Biotechnology Division, The Energy and Resources Institute (TERI), Habitat Place, Darbari Seth Block, Lodhi Road, New Delhi 110003, India, Tel: +91 11 24682100; Fax:+91 11 24682144; Email: ssubudhi@teri.res.in (or) sanjuktasubudhi@yahoo.com

\begin{abstract}
Currently there is an increased global interest on implication of nutritious diet on health sustainability. On this aspect nutraceuticals have assumed paramount importance based on their presumed safety and potential nutritional and therapeutic value. Microalgae are rich source of bioactive compounds having nutritional and therapeutic value and thus have found commercial applications as natural source of high value compounds. High value bioactive compounds from microalgae include: carotenoids, chlorophyll, polysaccharides, long chain polyunsaturated fatty acids, astaxanthin, antioxidants, micro and macronutrients, vitamins including several antimicrobial and antiviral compounds. Besides, some bioactive compounds from microalgae possess significant anti-inflammatory and antitumor activity. Potential of microalgae to produce such therapeutic compounds along with its simple growth requirement make them attractive from biotechnological perspective. Some of the biotechnologically relevant microalgal are Chlorella, Spirulina, Dunaliella, and Haematococcus. Though microalgae are known to human beings since decades, its potential biotechnological value is realized only in recent past. Microalgae still remain largely unexplored and urge for extensive investigation to explore for isolation and development of novel high value molecules. The present review highlights therapeutic and nutritional value of algal based bioactive compounds and also focuses on gaps and opportunities which should be exploited in the near future to evaluate the potential of microalgae as a novel source of health promoting compounds.
\end{abstract}

Received date: August 20, 2017

Accepted date: September 07, 2017

Published date: September 19, 2017

Citation: Sanjukta Subudhi. Bioprospecting for Algal Based Nutraceuticals and High Value Added Compounds. (2017) J Pharm Pharmaceutics 4(2): 145- 150.

DOI: $10.15436 / 2377-1313.17 .1651$

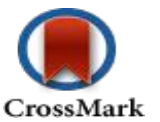

\section{Introduction}

For millennia, diverse microorganisms have yielded important biological materials useful to humans such as antibiotics, drugs, enzymes, herbicides, growth promoters and source of energy. Moreover, microbial diversity is the key to human survival and economic well-being and provides a huge reservoir of resources which we can utilize for our benefit. Despite the acknowledged value of microorganisms, our knowledge of their diversity and many of their key roles in sustaining global life support systems is still very scarce. The biological diversity of the Indian subcontinent is one of the richest in the world owing to its vast geographic area, varied topography and climate, and the juxtaposition of several bio geographical regions. Because of its richness in overall species diversity, India is recognized as one of the 12 mega diversity regions of the world. Chilika Lake of Orissa is one of the major mega-diversity centres of India. Lying on the eastern coast of Orissa, Chilika Lake is one of the largest lakes not only in India, but also in Asia and stretches over an area of $1,100 \mathrm{sq}$. $\mathrm{km}$. In fact, Chilika is the largest brackish water lake in Asia and second largest lake in the world and inhabits a mix of estuarine, marine and fresh water ecosystem. Large diversity of algae thrives in this habitat, which enhances the possibility of isolating novel algal strains having potential 
for production of nutraceuticals including high value bioactive compounds.

Recent decades have witnessed an increasing interest in nutraceuticals due to their significant therapeutic value which aid in providing human health benefits.

\section{Nutraceuticals: Significance in human health benefit}

With the emergence of industrialization, lifestyles of human beings have dramatically changed. Food habit is the first victim of this life style. Increasing work pressure, longer work schedules and various psychological pressures including increased spending capacity with rise in economy, have imposed people to develop fast eating food habits. Moreover, consumption of junk food has increased several manifolds that have led to a number of diseases related to nutritional deficiencies. These diseases include; diabetes, obesity, various cancers, vascular diseases and physiological disorder including degenerative diseases. Increasing concern for health care have dramatically raised the cost of medical care. Over the time people have realized that for maintaining normal functioning of human health, it is important to consume various foods rich in adequate nutrients. With the rise in health care cost, aging population, increasing awareness about fitness and health prompted people globally to lead healthier life by consuming functional foods rich in health enhancing compounds or 'nutraceuticals'. This development, in turn, is propelling expansion in the nutraceutical markets globally (Zhao, 2007, Pandey, 2010) $)^{[1,2]}$. Apart from this, scientific studies have also extended to these areas and provided supports to nutraceuticals and therapeutic services.

The term nutraceutical was coined from 'nutrition' and 'pharmaceutical' by Dr. Stephen De Felice in 1989. 'Nutraceutical' refer to natural functional/medical foods or bioactive compounds that have health promoting, disease preventing or medicinal properties and thus provide an opportunity to improve the human health. Considering these facts, nutraceuticals have received considerable global interest. The potential of nutraceuticals to accomplish desirable therapeutic outcomes with comparatively (compared to other therapeutic agents) reduced side effects has met with great monetary success (Nelson, 1999, Whiteman, 2001 ${ }^{[3,4]}$. Thus the preference for discovery and production of nutraceuticals is well appreciated by pharmaceutical and biotechnology industries.

Nutraceuticals help in providing protection against many diseases by acting as anti-obesity agents, cardiovascular agents, anticancer agents, anti-diabetic agent and immune boosters. Nutraceuticals include a variety of products like mineral and vitamin supplements, antioxidant supplements, probiotics, herbs, essential oils, fortified foods, health drinks and enzymes. Based on their functional properties, nutraceuticals are categorized into: functional foods, dietary supplements, medicinal food, botanicals and pharmaceuticals (Kokate, 2002, www.nutriforce. $(\mathrm{com}){ }^{[5]}$. Recently, nutraceuticals are receiving global recognition as being beneficial in treating coronary heart disease, obesity, diabetes, cancer, osteoporosis and other chronic and degenerative diseases like Parkinson's and Alzheimer's diseases. Mechanistic actions of nutraceuticals involve an array of biological processes that include activation of antioxidant defences, signal transduction pathways, cell survival-associated gene expression, cell proliferation, differentiation and preservation of mitochondrial integrity. These properties impart a crucial role in the protec- tion against pathologies of various age-related chronic diseases (Mandel et al., 2005) ${ }^{[6]}$.

\section{Nutraceuticals: Global market trend}

Rising global concern for health and nutrition including scientific evidence supporting health foods, are major factors that drive the worldwide nutraceuticals market. Main segments of nutraceutical market include: functional foods, dietary supplements, natural/herbal foods (Rishi, 2006) ${ }^{[7]}$. Dietary supplements segment is fast approaching maturity levels, particularly in developed regions. As per the BCC Research the global nutraceuticals market grew to $\$ 46.7$ billion in 2002 , at an AAGR (Annual Average Growth Rate) of nearly 7\%. In 2007 nutraceuticals sale is projected to reach $\$ 74.7$ billion at an AAGR of 9.9\% (www.bccresearch.com). The global nutraceuticals market is estimated at about $\$ 151$ billion in 2011. By 2016, it is estimated to reach nearly $\$ 207$ billion, a projected Compound Annual Growth Rate (CAGR) of 6.5\% between 2011 and 2016 (www. campanies and markets.com).

The present global scenario concerning the demand for nutraceuticals definitely urges for the need to focus research on exploration of robust microbial resources having potential for production of nutraceuticals and high value for the future benefit of mankind.

\section{Microalgae: As source of 'nutraceuticals' and 'high-value compounds'}

Microalgae are one of the potential organisms and useful to mankind in various ways and represent most promising resource for new products and applications (Pulz and Gross, $2004)^{[8]}$. Owing to their diverse chemical properties, microalgae can act as a nutritional supplement as well as a source of natural food colorants (Borowitzka, 1999, Soletto et al., 2005, Apt and Bahrens, 1999) ${ }^{[9-11]}$. They are an enormous biological resource and are cultivated as a source of several bioactive high value compounds like, polyunsaturated fatty acids, carotenoids, phycobilliproteins, polyols, polysaccharides, antioxidants. These high value compounds possess antimicrobial, antiviral, antioxidant, anti-inflammatory, anti-cancer, anti-fungal activity and some act as immune modulator.

Though some microalgae like, Nostoc and Arthrospira, have been exploited for millennia, commercial potential of microalgae is recognized only in recent past. Among 30,000 species that are believed to exist (Chaumont, 1993, Radmer and Parker, 1994) ${ }^{[12,13]}$ only a few hundred are investigated for chemical content out of which very few are cultivated in industrial grade (Olaizola, 2003). Recently, microalgae biomass has been exclusively used in health food market and its commercial potential is widely recognized. The growing use of algal biomass for nutraceutical purposes is expected to provide an attractive revenue stream for algal industries.

During last decade, micro algal biotechnology has gained considerable attention owing to their substantially higher productivity compared to traditional crops including their potential to grow in areas and climate that are not suitable for agricultural crops. Further, microalgae production serves as an important natural mechanism to reduce atmospheric carbon $\mathrm{CO}_{2}$ by bio fixation and helps in lowering the greenhouse effect. In view of this, the application of micro algal biomass and/or metabolites offers a promising and innovative approach for the development 
of healthier food products.

Most biotechnologically relevant microalgae include species of green algae (Chlorophyceae): Chlorella vulgaris, Haematococcuspluvialis, Dunaliellasalina and species of cyanobacteria (blue green algae): Spirulina maxima and Spirulina platensis (Gouveia et al., 2008, Guedes et al., 2011) ${ }^{[14,15]}$.

However, microalgae especially of marine origin still remain to date largely unexplored and represent a unique opportunity to explore novel metabolites and production of cost effective known metabolites. Marine based compounds known to entail a wide diversity of molecular targets with a marked selectivity, which raises pharmaceutical interest (Guedes et al., 2011) $)^{[15]}$.

\section{Algal based bioactive compounds: Potential nutritional and therapeutic value}

Primary and secondary metabolites that are synthesized by microalgae and are currently being commercialized or under consideration for commercial extraction include: chlorophylls, carotenoids, phycobilins, long chain polysaccharides, vitamins and minerals, tocopherols and sterols, proteins, $\beta$-carotene, astaxanthin, allophycocyanin. These bioactive compounds serve as antioxidants, anti-inflammatory agents, antimicrobial agent, antiviral agent and antitumoral agent, based on their specific activity (Borowitzka, 1999, Apt and Bahrens, 1999) ${ }^{[9,11]}$.

\section{Chlorophylls}

Chlorophyll-a present is the primary photosynthetic pigment and present in all algae. Chlorophyll-b present in species belonging to Chlorophyta and Euglenophyta group. However, several marine algae possess chlorophyll-c, -d and -e. Chlorophylls have got significant application in food and pharmaceutical colorants and in traditional medicine owing to its healing and anti-inflammatory properties. It also helps in reducing the risk of colorectal cancer (Balder et al., 2006 ${ }^{[16]}$. Oxidative stress and inflammation are implicated in several health conditions like atherosclerosis and cardiovascular diseases.

\section{Carotenoids}

Carotenoids are naturally occurring fat-soluble pigments found principally in algae and play critical role as photoprotective agent. Carotenoids are potential biological antioxidants which are able to absorb the excitation energy of Single Oxygen Radicals (SOR) into their complex ringed chain and helps in lowering tissue decay by reducing the DNA, protein and membrane lipid damage. In human beings, carotenoids can serve several important functions. Consumption of carotenoid rich diet helps in lowering the risk for several diseases including arteriosclerosis, cataracts, age-related macular degeneration, cancer, multiple sclerosis (Tapiero et al., 2004) ${ }^{[17]}$. About 50 carotenoids have provitamin-A activity which include; $\alpha$-carotene, $\beta$-carotene and $\beta$-cryptoxanthin (Faure et al., 1999). Among all, $\beta$-carotene and astaxanthin are attractive algal products and have high market demand. Presently, $\beta$-carotene is mainly derived from Dunaliellasalina and is used extensively as a yellow-orange food colouring and as anticancer agent (Plaza et al., 2009) [18].

Currently, astaxanthin is produced from Haematochoccuspluvialis (Plaza et al., 2009) ${ }^{[18]}$. Consumption of astaxanthin helps in enhancing eye health, muscle strength, protecting skin from premature ageing, inflammation, protects from cancer by preventing certain tumor growth. Astaxanthin also provides protection against oxidation of essential polyunsaturated fatty acids. Free radical fighting capacity of astaxanthin known to be 500 times more efficient as compared that of vitamin E (Dufoss et al., $2005)^{[19]}$. Dietary astaxanthin also helps in reducing low-density lipoprotein oxidation and is safe for human use (Iwamoto et al., 2000, Spiller and Dewell, 2003 ${ }^{[20,21]}$. Further, outstanding antitumoral activity of astaxanthin has attracted significant global interest in large scale production of this bioactive compound. Antitumoral effect of dietary astaxanthin is displayed in the post-initiation phase of carcinogen-induced colon and oral cancer models (Tanaka et al., 1995) ${ }^{[22]}$. In addition, acetylenic carotenoids like: diatoxanthin, heteroxanthin, diadinoxanthin, isolated from microalgae exhibit antitumoral properties (Dembitsky, $2006)^{[23]}$. Significant source of acetylenic carotenoids include: Euglenaviridis, Tribonemaaequala, Gonyostomium semen, Vacuolariavirescens, Pleurochlorismeiringensis (Oono et al., 1995) [24].

Lutein, another carotenoid pigment produced from Chlorella pyrenoidosa helps in preventing cancer and diseases related to retinal degeneration (Cha et al., 2008) ${ }^{[25]}$.

\section{Phycobiliproteins (phycocyanin, phycoerythrin)}

Phycobiliproteins are deep coloured water soluble fluorescent pigments. Algal species belonging to Cyanobacteria, Rhodophyta and Cryptomonad group encompass these pigments. Phycobiliproteins have got significant applications and are mainly used as highly sensitive fluorescent markers in clinical diagnosis as well as in labelling antibodies. Phycocyanin and phycoerithrin are major pigments which belong to this group. Phycocyanin is blue, water soluble, light-harvesting pigment and found in cyanobacteria. Phycocyanin is strongly fluorescent and mainly used as natural colouring in chewing gums, ice creams, candies, jellies, dairy products including in cosmetics (Sekar and Chandramohan, 2007) ${ }^{[26]}$. Apart from these, phycocyanin also serves as excellent antioxidant, anti-inflammatory agent (Eriksen, 2008, Romay et al., 2003) $)^{[27,28]}$.

\section{Omega-3 (n-3) and omega-6 (n-6) Poly Unsaturated Fatty acids (PUFA)}

Fatty acids from microalgae are generally rich in Poly Unsaturated Fatty Acids (PUFA) and have a higher degree of unsaturation. PUFA are Essential Fatty Acids (EFA) that is required for proper functioning of cells. Human body cannot synthesize PUFA and hence requires dietary supplementation of PUFA for maintaining good health. Dietary deficiency in long chain n-3 polyunsaturated fatty acids is firmly associated with increased morbidity and mortality from coronary heart disease (Simopoulos, 2002) $)^{[29]}$.

Microalgae have potential to synthesize PUFAs of both omega-3 and omega-6 series. Long chain PUFAs contains straight chain carboxylic acids of 20 or more carbon atoms that contain 3 or more double bonds. Omega-3 PUFAs include: Eicopentaenoic acid (EPA) and Docosahexaenoic acid (DHA). Recently, algal based omega-3 (n-3) PUFAs got considerable attention due to their association with the prevention and treatment of several diseases like, atherosclerosis, thrombosis, arthritis and cancer, blood pressure, coronary heart disease, rheu-

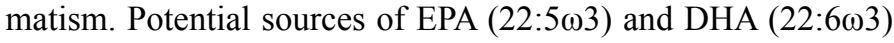


include: Nannochloropsis, Phaedactylum, Nitzschia, Isochrysis, Diacronema and Crypthecodinium, Schizochytrim, respectively (Simopoulos, 2002) $^{[29]}$.

Omega-6 PUFAs include, gamma linolenic acid (GLA, $18: 3 w 6)$. GLA is an 18-carbon polyunsaturated fatty acid that contains three double bonds. Sources of GLA are limited and include only few species of plant like, borage, hemp, evening primrose, black current, Echium including fungal oil (Subudhi et al., 2008). However, demand of GLA is increasing as human body cannot synthesize this PUFA and needs dietary supplement. Microalgae especially the cyanobacterium 'Spirulina' is rich in GLA and thus serves as an attractive source of 'GLA'. Dietary GLA plays key role in prevention and treatment of several diseases like, blood pressure, atherosclerosis, cardiac arrhythmia, prostate cancer, glioma, and liver cancer, auto immune diseases like acute respiratory distress syndrome, rheumatoid arthritis, skin diseases, and diabetes including premenstrual syndrome. GLA also helps in boosting immunity and helps in proper brain functioning (Rakesh and Harikumar, 2005).

\section{Vitamins and Minerals}

Microalgae represent a significant source of essential vitamins $\mathrm{A}, \mathrm{B}_{1}, \mathrm{~B}_{2}, \mathrm{~B}_{6}, \mathrm{~B}_{12}, \mathrm{C}, \mathrm{E}$, nicotinate, biotin, folic acid and pantothenic acid and minerals, $\mathrm{Na}, \mathrm{K}, \mathrm{Ca}, \mathrm{Mg}, \mathrm{Fe}, \mathrm{Zn}$ including trace minerals (Borowitzka, 1988) ${ }^{[31]}$.

\section{Polysaccharides}

Polysaccharides have got significant implications in food industry as thickening agents. Commercial polysaccharides include, agar, alginates, carrageenans from Laminaria, Gracilaria, Macrocystis. Sulphated polysaccharides from Porphyridium species contain 10 sugars in addition to glycoproteins and inorganic sulphate, which aids in inhibiting the spreading of immune cell recruitment toward inflammatory stimuli in vivo (Matsui et al., 2003 $)^{[32]}$. In addition, this polysaccharide also inhibits auto-oxidation of linoleic acid and other forms of oxidative damage in a dose dependent manner and helps in stimulating human immune system (Pulz and Gross, 2004) ${ }^{[33]}$. Extracellular polysaccharide extracts from Chlamydomonas mexicana have found application as a soil conditioner (Borowitzka, 1988) ${ }^{[31]}$.

\section{Antiviral compounds}

In recent years, many infectious diseases have emerged that urge for exploration of novel antiviral compounds. In view of this, several antiviral drugs have been specifically developed. However, drug-resistance mutations take place constantly, which raises serious concern for development of novel antiviral compounds with desired properties. Microalgae represent a large reservoir for novel compounds and thus have received global attention as potential source of antiviral agents (Borowitzka, 1995 $)^{[34]}$. Bioactive compound, pheophorbide-a,-b like compound extracted from Dunaliellaprimolecta exhibit antiviral activity and helps in acting against HSV1 virus by inhibiting cytopathic effect (Ohta et al., 1998) ${ }^{[35]}$. Allophycocyanin extracts from Cryptomonad algal group have profound antiviral activity against Enterovirus 71. Allophycocyanin acts by inhibiting cytopathic effect and delays viral RNA synthesis (Shih et al., $2000)^{[36]}$. A homopolysaccharide of galactose with uronic acid and sulphate groups from Gyrodiniumimpudicum strain exhibit an impressive activity in vitro against swine Encephalomycardi- tis virus, widely found at clinical level (Yim et al., 2004) $)^{[37]}$.

\section{Antimicrobial compounds}

Global concern for development of novel antibiotic compounds increased consequently with the emergence of multidrug resistant bacterial strains like Staphylococcus aureus. It is imperative to explore novel antibacterial compounds in order to overcome the multidrug resistance problem. Marine microalgae serve as unexplored reservoir for novel antimicrobial compounds.

Short chain fatty acids from Haematococcuspluvialis and organic extracts from Euglena viridis, possess significant antibacterial activity and act against Vibrio species and Pseudomonas species, respectively. Methanolic acid extracts of Chlamydomonasreinhardtii exhibit antifungal activity and act against Staphylococcus aureus.

\section{Future Prospects}

Nutraceuticals are currently receiving global recognition due to their significant nutritional and therapeutic value. Microalgae have exceptional nutraceutical and therapeutic value and thus offer as a promising biological resource to explore in future for utilization in food, feed, cosmetic and pharmaceutical industries. Considering these facts, during last decade microalgae have gained importance in industrial scale for production of nutraceuticals. In addition, other advantage of microalgae is their fast growth rate and potential to attend high cell density at optimum condition.

The acceptance of use of microalgae biomass and their bio molecules has resulted in development of many innovative food products in recent past. However, from biotechnology perspective, microalgae are far behind and needs extensive investigation for the benefit of mankind. Several secondary metabolites from microalgae have shown to possess potential therapeutic value. These results point to promising future development of microalgal biotechnology. Among 10,000 species that are identified only few are investigated to date for their potential chemical content and very few are cultivated in industrial scale. Other species need comprehensive screening for human health benefit. In the recent past, microalgae-derived product market is steadily expanding. But its substantial growth is hampered by the manufacture technology used. Major challenge is cost effective production and optimized recover operation.

The overall competitiveness of microalgae based bioprocesses entails improved performance of microalgae via genetic engineering, metabolic engineering, process engineering, strain improvement for faster growth and higher yield including comprehensive screening of existing micro algal species for novel bioactive compounds as well as isolation of novel robust micro algal species having potential for production of novel bioactive compounds. Especially marine microalgae remain largely explored and needs extensive investigation to explore for isolation of novel bioactive compounds.

\section{Acknowledgement}

The authors sincerely thank Director, Environmental and Industrial Biotechnology Division and Director General, The Energy \& Resources Institute (TERI), New Delhi, India for the kind support and for providing infrastructure facilities to carry out this work. Authors are grateful to the, Department 
of Science and Technology, Government of India for financial assistance for the work.

\section{References}

1) Zhao, J. Nutraceuticals, nutritional therapy, phytonutrients, and phytotherapy for improvement of human health: A perspective on plant biotechnology application. (2007) Recent Pat Biotechnol 1(1): 75-97. Pubmed |Crossref |Others

2) Pandey, M., Verma, R.K., Saraf, S.A. Nutraceuticals: new era of medicine and health. (2002) Asian J Pharmaceutical and Clinical Res 3(1): 11-15.

Pubmed $\mid$ Crossref $\mid$ Others

3) Nelson, N.J. Purple carrots, margarine laced with wood pulp? Nutraceuticals move into the supermarket. (1999) J Natl Cancer Inst 91(9): 755-757.

Pubmed | Crossref | Others

4) Whitman, M. Understanding the perceived need for complementary and alternative nutraceuticals: lifestyle issues. (2001) Clin J Oncol Nurs 5(5): 190-194.

Pubmed | Crossref $\mid$ Others

5) Kokate, C.K., Purohit, A.P., Gokhale, S.B. Nutraceutical and Cosmaceutical. (2002) Pharmacognosy, 21st edition, Pune, India 542-549. Pubmed | Crossref | Others

6) Mandel, S., Packer, L., Youdim, M.B., et al. Proceedings from the Third International Conference on Mechanism of Action of Nutraceuticals. (2005) J Nutritional Biochem 16(9): 513-520.

Pubmed | Crossref $\mid$ Others

7) Rishi, R.K. Nutraceuticals: Borderline between food and drugs. (2006) Pharma Review 2006.

Pubmed | Crossref $\mid$ Others

8) Pulz, O., Gross, W. Valuable products from biotechnology of microalgae. (2004) Appl Microbiol Biotechnol 65(6): 635-648.

Pubmed | Crossref | Others

9) Borowitzka, M.A. Commercial production of microalgae: ponds, tanks, tubes and fermenters. (1999) J Biotechnol 70(1-3): 313-321.

Pubmed | Crossref $\mid$ Others

10) Soletto, D., Binaghi, L., Lodi, A., et al. Batch and fedbatch cultivations of Spirulina platensis using ammonium sulphate and urea as nitrogen sources. (2005) Aquaculture 243(1-4): 217-224.

Pubmed | Crossref $\mid$ Others

11) Apt, K.E., Behrens, P.W. Commercial developments in microalgal biotechnology. (1999) J Phycol 35(2): 215-226.

Pubmed | Crossref $\mid$ Others

12) Chaumont, D. Biotechnology of algal biomass production: a review of systems for outdoor mass culture. (1993) J Appl Phycol 5(6): $593-$ 604.

Pubmed | Crossref | Others

13) Radmer, R.J., Parker, B.C. Commercial applications of algae: opportunities and constraints. (1994) J Appl Phycol 6(2): 93-98.

Pubmed $\mid$ Crossref $\mid$ Others

14) Gouvia L, Batista A.P., Sousa I, et al. Microalgae in novel food products. (2008) In: food Chemistry Research Developments, Nova Science Publishers, 75-112.

Pubmed | Crossref $\mid$ Others

15) Guedes, A.C., Amaro, H.M., Malcata, F.X. Microalgae as source of high added value compounds- a brief review of recent work. (2011) Biotechnol Prog 27(3): 597-613.

Pubmed | Crossref|Others

16) Balder, H.F., Vogel, J., Jansen, M.C., et al. Heme and chlorophyll intake and risk of colorectal cancer in the Netherlands cohort study. (2006) Cancer Epidemiol Biomarkers Prev 15(4): 717-725.

Pubmed | Crossref | Others
17) Tapiero, H., Townsend, D.M., Tew, K.D. The role of carotenoids in the prevention of human pathologies. (2004) Biomed Pharmacother 58(2): 100-110.

Pubmed | Crossref | Others

18) Plaza, M., Herrero, M., Cifuentes, A., et al. Innovative natural functional ingredients from microalgae. (2009) J Agric Food Chem 57(16): 7159-7170.

Pubmed | Crossref | Others

19) Dufossé, L., Galaup, P., Yaron, A., et al. Microorganisms and microalgae as sources of pigments for food use: a scientific oddity or an industrial reality?. (2005) Trends in Food Science and Technology 16(9): 389-406.

Pubmed | Crossref $\mid$ Others

20) Iwamoto, T., Hosoda, K., Hirano, R., et al. Inhibition of low density lipoprotein oxidation by astaxanthin. (2000) J Atheroscler Thromb 7(4): 216-222.

Pubmed | Crossref|Others

21) Spiller, G.A., Dewell, A. Safety of an astaxanthin-rich Haematococcus pluvialis algal extract: a randomized clinical trial. (2003) J Med Food 6(1): 51-56.

Pubmed | Crossref | Others

22) Tanaka, T., Kawamori, T., Ohnishi, M., et al. Suppression of azoxymethane-induced rat colon carcinogenesis by dietary administration of naturally occurring xanthophylls, astaxanthin and canthaxanthin, during the postinitiation phase. (1995) Carcinogenesis 16(12): 2957-2963.

Pubmed | Crossref| Others

23) Dembitsky, V.M. Anticancer activity of natural and synthetic acetylenic lipids. (2006) Lipids 41(10): 883-924.

Pubmed | Crossref | Others

24) Oono, M., Kikuchi, K., Oonishi, S., et al. Anticancer Agents Containing Carotenoids. (1995) Japan Kokai, TokkyoKoho. 5.

Pubmed $\mid$ Crossref $\mid$ Others

25) Cha, K.H., Koo, S.Y., Lee, D.U. Antiproliferative effects of carotenoids extracts from Chlorella ellipsoidea and Chlorella vulgaris on human colon cancer cells. (2008) J Agric Food Chem 56(22): 1052110526.

\section{Pubmed | Crossref | Others}

26) Sekar, S., Chandramohan, M. Phycobiliproteins as a commodity: trends in applied research, patents and commercialization. (2008) J Appl Phycol 20(2): 113-136.

Pubmed | Crossref | Others

27) Eriksen, N.T. Production of Phycocyanin - a pigment with applications in biology, biotechnology, foods and medicine. (2008) Appl Microbiol Biotechnol 80(1): 1-14.

Pubmed | Crossref| Others

28) Romay, C.H., Gonzalez, R., Ledon, N., et al. Phycocyanin: a biliprotein with antioxidant, anti-inflammatory and neuroprotective effects. (2003) Curr Protein and Pept Sci 4(3): 207-216.

Pubmed | Crossref | Others

29) Simopoulos, A.P. The importance of the ratio of omega-6/omega-3 essential fatty acids. (2002) Biomed Pharmacother 56(8): 365-379.

Pubmed | Crossref| Others

30) Kapoor, R., Nair, H. Gamma linolenic acid rich oils. (2005) Sixth Edition. Bailey's Industrial Oil and Fat Products.

Pubmed | Crossref | Others

31) Borowitzka, M.A., Borowitzka, M., Borowitzka, L., et al. Vitamins and fine chemicals from micro-algae. (1988) In M.A 153-196.

Pubmed | Crossref | Others

32) Matsui, M.S., Muizziddin, N., Arad, S., et al. Sulfated polysaccharides from red microalgae have antiinflammatory properties in vitro and in vivo. (2003) Appl Biochem Biotechnol 104(1): 13-22.

Pubmed | Crossref | Others

33) Pulz, O., Gross, W. Valuable products from biotechnology of microalgae. (2004) Appl Microbiol Biotechnol 65(6): 635-648.

Pubmed | Crossref| Others 
34) Borowitzka, M.A. Microalgae as sources of pharmaceuticals and other biologically active compounds. (1995) J Appl Phycol 7(1): 3-15. Pubmed |Crossref $\mid$ Others

35) Ohta, S., Ono, F., Shiomi, Y., et al. Anti-herpes simplex virus substances produced by the marine green alga, Dunaliella primolecta. (1998) J Appl Phys 10(4): 349-356.

Pubmed|Crossref|Others

36) Shih, S.R., Ho, M.S., Lin, K.H., et al. Genetic analysis of Enterovirus 71 isolated from fatal and non-fatal cases of hand, foot and mouth disease during an epidemic in Taiwan, 1998. (2000) Virus Res 68(2): 127-136.

Pubmed | Crossref $\mid$ Others

37) Yim, J.H., Kim, S.J., Ahn, S.H., et al. Antiviral effects of sulphated exopolysacchride from the marine microalga Gyrodinium impudicum strain KG03. (2004) Mar Biotechnol (NY) 6(1): 17-25.

Pubmed | Crossref | Others

38) Borowitzka, M.A., Borowitzka, L.J. Micro-algal biotechnology. (1990) Cambridge, UK: Cambridge University Press. 47(2): 181-182.

Pubmed |Crossref | Others

39) GA-085R. Evolving nutraceutical.

Pubmed | Crossref | Others

40) Nutiforce.com (nutraceuticals for health).

Pubmed | Crossref $\mid$ Others 